\title{
SIMILARITY BASED ClassificATION AND DETECTION OF RESPIRATORY STATUS IN FREQUENCY DOMAIN
}

\author{
Suyeol Kim ${ }^{1}$, Chaehwan Hwang ${ }^{1}$, Jisu Kim² ${ }^{2}$ Cheolhyeong Park ${ }^{2}$ and \\ Deokwoo Lee ${ }^{2}$ \\ ${ }^{1}$ Department of Biomedical Engineering, Keimyung University, Daegu, \\ Republic of Korea \\ ${ }^{2}$ Department of Computer Engineering, Keimyung University, Daegu, Republic \\ of Korea
}

\begin{abstract}
Sleep apnea is considered one of the most critical problems of human health, and it is also considered one of the most important bio-signals in the area of medicine. In this paper, we propose the approach to detection and classification of respiratory status based on cross correlation between normal respiration and apnea, and on the characteristics of respiratory signals. The characteristics of the signals are extracted by analyzing frequency analysis. The proposed method is simple and straightforward so that it can be workable in practice. To substantiate the proposed algorithm, the experimental results are provided.
\end{abstract}

\section{KEYWORDS}

Respiration, Apnea, Fourier transform, Detection, Classification

\section{INTRODUCTION}

Obstructive breathing disease can lead to severe health problems such as abnormal lung conditions (asthma, emphysema, etc.), heart disease, allergies, etc. Extensive research has been carried out to find methods for diagnosis, treatment or prevention. In the areas of signal analysis and processing, detection and monitoring of respiratory signal have long been of interest, and to achieve efficient measurement of respiration also has been extensively investigated [1]. Major methods of signal acquisition can be categorized in invasive or non-invasive medical instruments $[2,3]$. In general, vital signs (also called bio signal) have been able to play a key role to indicate the status of human health. Among the other signs, body temperature, heart rate, blood pressure, electrocardiogram and respiratory rate are considered the most primary vital signs [4]. Furthermore, recording the signs above is also considered the standard for monitoring the health status of patients in hospital ward. However, it has been reported that respiratory signal has gained less attention than the other three signals even though respiratory status can be both an important indicator and a predictor of severe illness [4]. Since a few years, there have been extensive research on detecting sleep apnea one of the breathing disorders. Sleep apnea can cause cardiovascular problem, so there have been research on detection and monitoring of the breathing status using invasive or non-invasive methods (or contact based or non-contact based methods). 
Contact based monitoring methods use devices that are attached to human body, but the methods undergo difficulties when the patients suffer from generating respiratory signal. Besides, the monitoring equipment is usually expensive, gives discomfort to testee people, and they need to be guided how to generate their respiratory signal that can sufficiently provide meaningful analysis results. However, the contact based method, if it is stable setup for use, acquired signal is reliable, leading to accurate measurement and analysis. On the other hand, non-contact or non-invasive method can be alternatives to the contact based methods. They can be audio based, temperature based or vision based or distance based (e.g., UWB radar) methods [5, 6]. In general, non-contact based devices are, in general, low cost and easy to use compared to the contact based ones. This paper chiefly focuses on classification of respiratory signal that is acquired using a contact-based medical instrument. This work aims at detecting a signal of an abnormal respiration. Abnormal respiration in this work is apnea. Respiratory signal is defined in 1-dimensional domain, and the classification is basically performed using correlation coefficient between the signals. The proposed approach is expected to maximize inter-class distance and to minimize intra-class distance. The overall description of the present work is shown in Figure. 1.

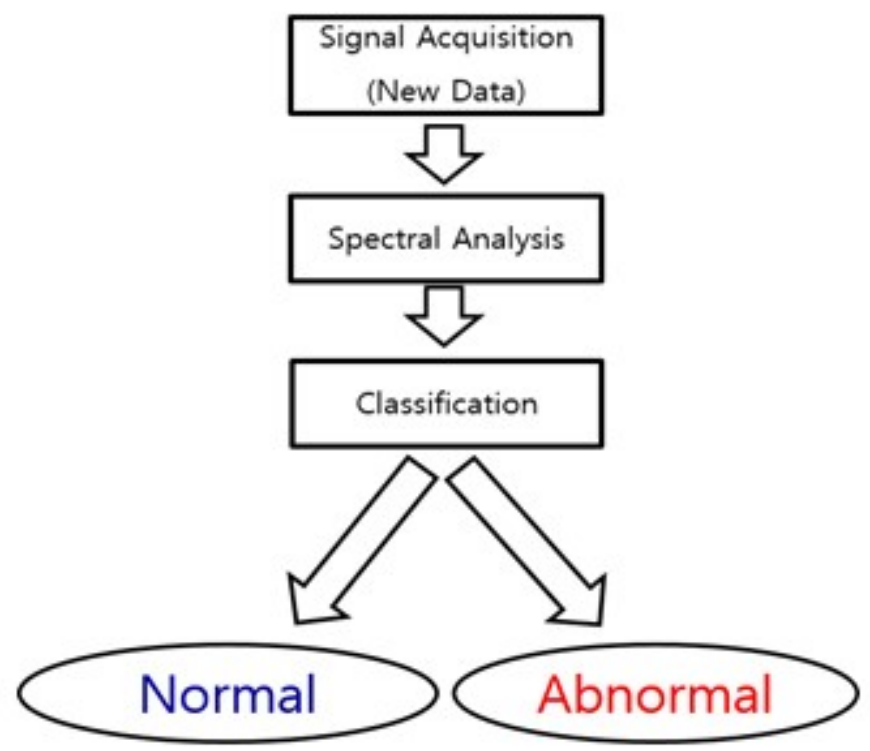

Figure 1. Overall architecture of a classification based monitoring and detection of respiratory status

Rest of the paper is organized as follows. In section II, preprocessing, noise reduction using Savitzky-Golay and median filter are introduced, and the filtering results are also briefly provided. In section III, similarity between the different respiratory signals are estimated so that classification of normal and abnormal status can be performed. Experimental results are provided in section IV, and we conclude this paper and suggest future direction.

\section{NOISE REDUCTION}

Respiratory signal is acquired using a UWB radar sensor, NOVELLDA X4, and the device is not only used for respiration measurement, but also for detection of dynamic activities. The measurement is based on the calculating the distance by estimating duration of reflection of radar 
signal. The device can provide denoised signal so that users do not have to endeavor to reduce or remove noise component. In case of existence of noise component, we have used a filter that combines Savitzky-Golay filter and median filter [7]. The filter not only reduces noise component but also maintains high frequency component of a signal. Savitzky-Golay (SG) filter has been used in the areas of signal denoising by smoothing the signal with noise of high frequency components. Let be an original signal (this signals is ideally noiseless signal), random noise is added to the signal. Let and be the denoised signal and the signal with noise, respectively, is represented as follows.

$$
x_{n}(t)=x(t)+n(t)
$$

and is random noise. The noise model is not discussed in this paper because it is beyond the scope of the present work. SG filtering process can be represented as an average of convoluted noised signal, and written as follows.

$$
x_{d}\left(t_{j}\right)=k \cdot \sum_{i=-M}^{M} C_{i} \cdot x_{n}\left(t_{j+i}\right)
$$

where $k$ is $\frac{1}{2 M+1}, \mathrm{a} \cdot b$ is an inner product of a and $b, 2 M+1$ is the smoothing window, $C_{i}$ is the smoothing coefficient (or convolution coefficient), respectively [7]. The essential idea behind SG filter is signal smoothing based on polynomial approximation using least-squares method. Due to the limitation of the SG filter, as explained in the next section, median filter is applied to the signal. Median filter, nonlinear filter, has been popularly used in image processing. Median filtering can be simply performed by extracting the median value in moving window. In the present work, since the signal of the interest is 1-dimension, the running window is simply a row vector (or column vector). The size (or length) of a vector can be adjusted according to the areas of applications. While the basic idea of median filter is very simple, it preserves high frequency components of a signal. Intuitively, median filter still show limitation in that it achieves successful preservation of high frequency components in certain conditions and loses information in boundary areas of a signal. Concerning all above, this paper proposes the new filter that combines SG filter, $h_{s g}(t)$ and median filter, $h_{m}(t)$. SG filter shows significant performance in smoothing signal, and median filter shows strength in preservation of edge information. This paper, hence, fully exploits both properties of the filters. The proposed filter, $h_{c}(t)$ is applied to the noised signal, and it reduces the noise components while preserves edge information, and $h_{c}(t)$ is written as

$$
x_{d}(t)=h_{c}(t) * x_{n}(t)
$$

where $h_{c}(t) * x_{n}(t)$ represents the convolution between $h(t)$ and $x_{n}(t)$, and $h_{c}(t)=h_{m}(t) *$ $h_{s g}(t) . h_{c}(t)$ is a composite filter of SG filter and median filter, and can be represented as

$$
x_{d}\left(t_{j}\right)=k \cdot \sum_{i=-M}^{M} C_{i} \cdot\left[h_{m}\left(t_{j+i}\right) * x_{n}\left(t_{j+i}\right)\right]
$$

Filtered respiration results are shown in Fig.s 5 and 6 [9]. 


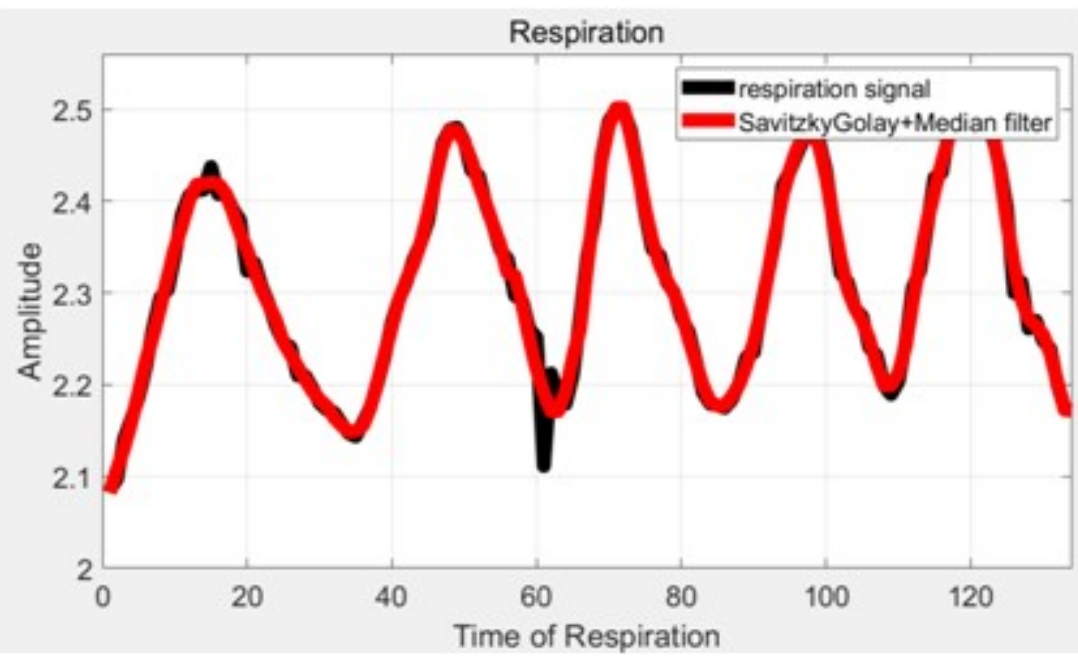

Figure 2. Filtered normal respiration signal



Figure 3. Filtered apnea signal

\section{DECiSion Of ReSPiRATORY Status}

In this paper, respiratory signal is analyzed in frequency domain. Since the acquired signal is defined in discrete time domain, discrete Fourier Transform (DFT) is used in this work.

$$
X[k]=\sum_{n=0}^{N-1} x[n] e^{-j \frac{2 \pi k n}{N}}, k=0,1, \ldots \ldots, N-1
$$

Once DFT is calculated, the result is composed of magnitude response and phase response. This paper chiefly deals with magnitude response. In particular, decision of respiratory status is based on the frequency component in which the magnitude response is maximized.

$$
\hat{f}=\operatorname{argmax}_{f}|X[k]|
$$


where $\hat{f}$ is a frequency component that corresponds to the maximum magnitude response. Once $\hat{f}$ is estimated, the function $o(\hat{f})$ determines the status of respiration.

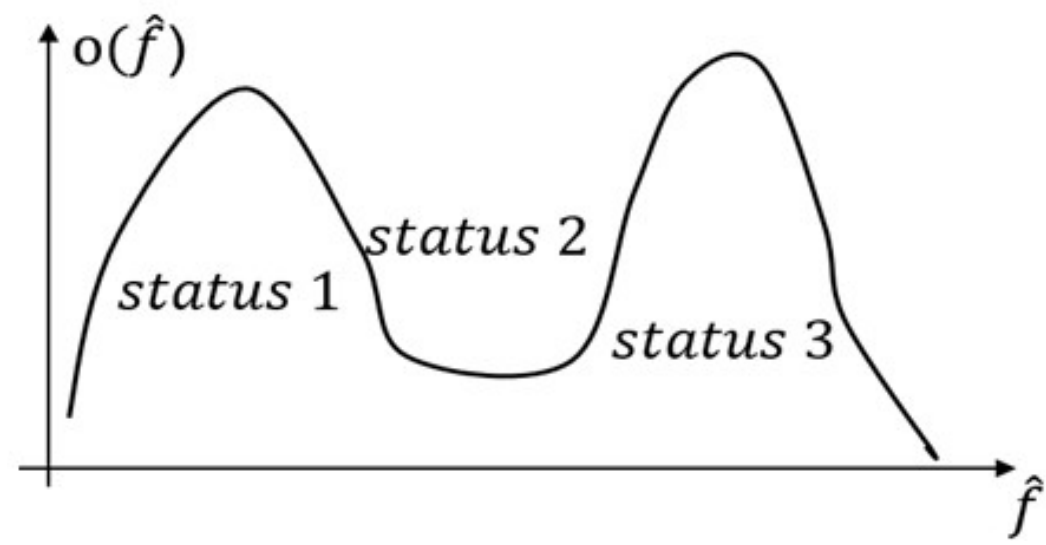

Figure 4. Decision function to estimate respiratory status

\section{EXPERIMENTAL RESUlts}

This section details the experimental results that substantiate the proposed approach. UWB radar sensor, NOVELLDA X4 is used. Respiration is categorized as normal and abnormal status. Normal signal is categorized as totally normal one and the normal status while speaking. Abnormal signal is an apnea which is partially contained in normal signal. Each person provides respiration signal composed of three status stated above. Each person provides 50 respiration signals each of which can be one of three statuses. Each respiration is acquired during about 60 seconds, and ages are between $23 \sim 25$. Apnea is contained in the normal signal, and it is generated during about 15 seconds. Sampling frequency of the signal is $10 \mathrm{~Hz}$. Examples of respiration signals are shown in Fig.s 5 - 7. Results of spectral analysis of respiratory statuses are shown in Fig.s 8 - 10

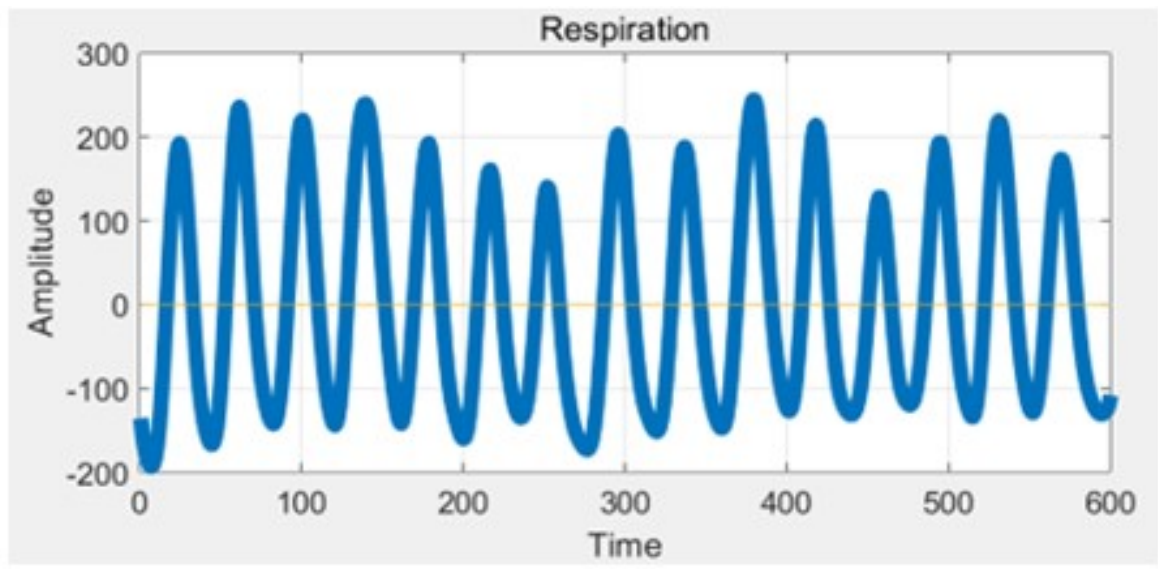

Figure 5. Totally normal respiration 


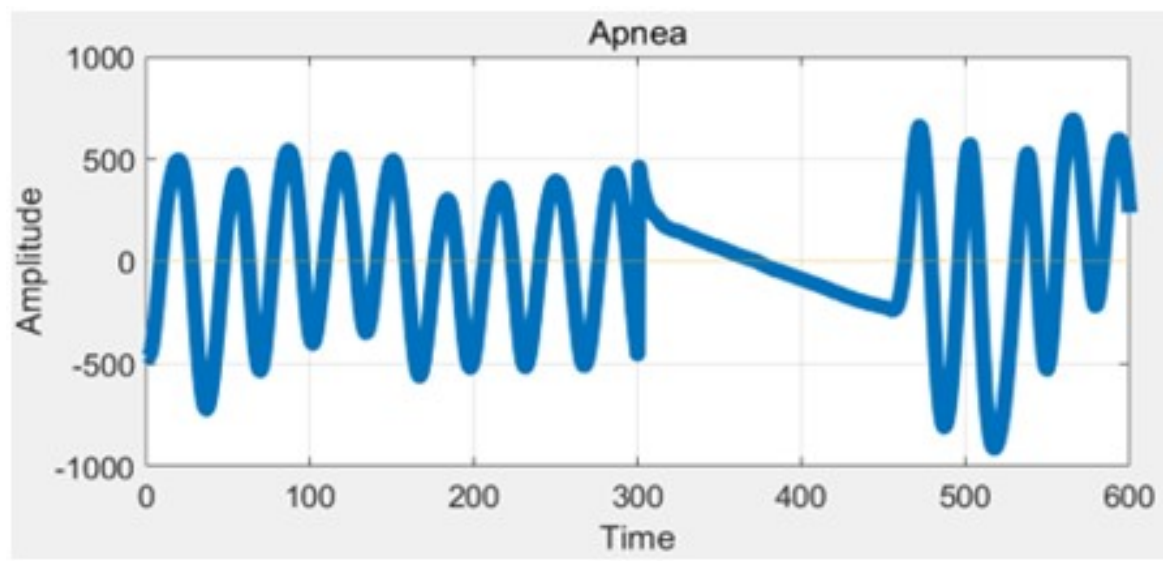

Figure. 6. Partial apnea contained in normal respiration

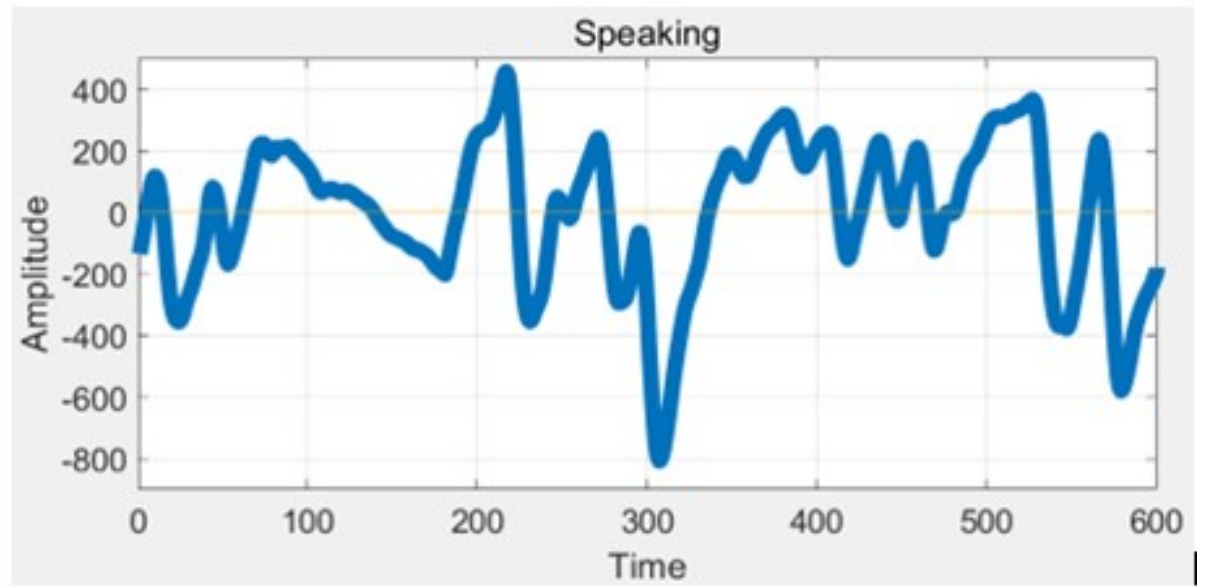

Figure. 7. Normal respiration during speaking activity

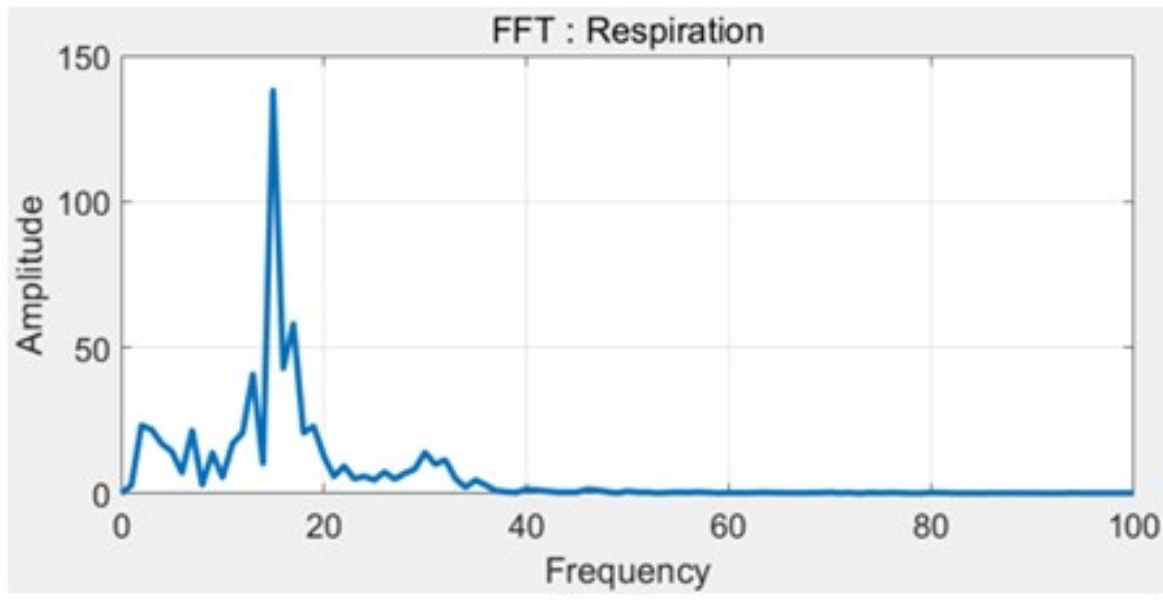

Figure. 8. Magnitude response of DFT of normal respiration 


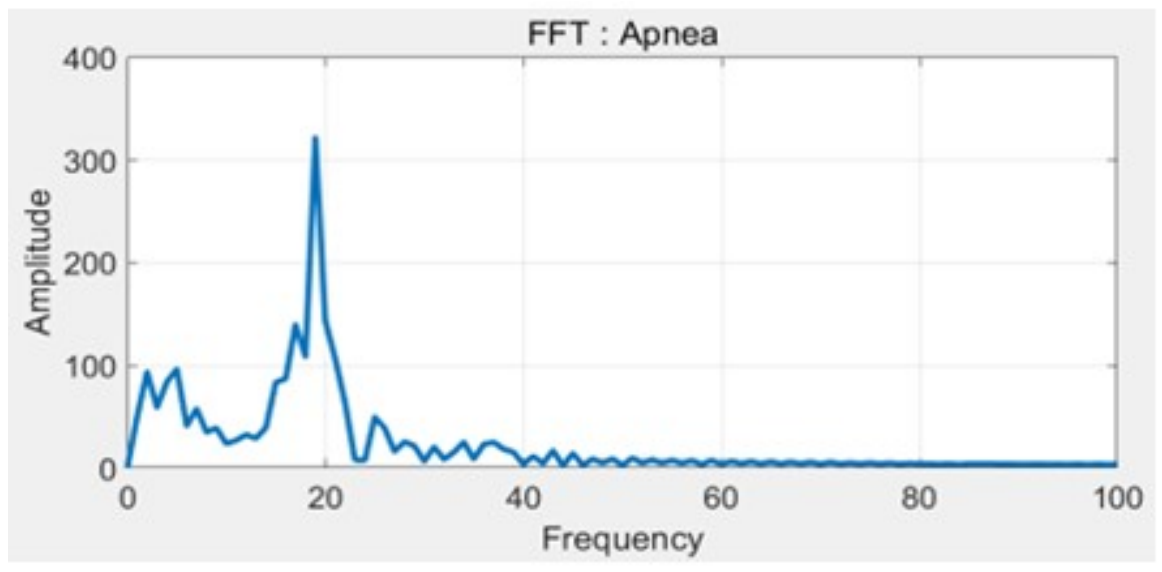

Figure. 9. Magnitude response of DFT of apnea signal

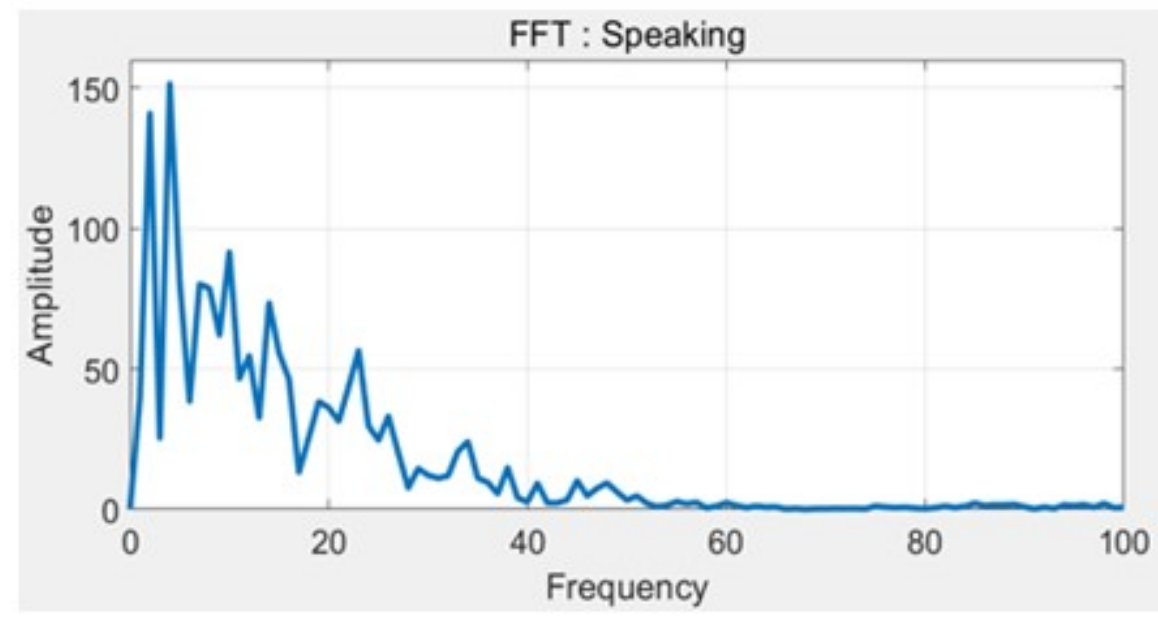

Figure. 10. Magnitude response of DFT of normal respiration with speaking activity

Frequency component that corresponds to the maximum magnitude response is estimated to classify the respiratory status. As shown in Fig.s 8-10, can be used to classify respiratory status, e.g., is in the range of $11-16 \mathrm{~Hz}$ (average is $13.2 \mathrm{~Hz}$ ), $17-27 \mathrm{~Hz}$ (average is $20.9 \mathrm{~Hz}$ ) and $2-$ $4 \mathrm{~Hz}$ (average is $3 \mathrm{~Hz}$ ), in case of normal respiration, apnea and normal respiration during speaking activity, respectively. Intuitively, apnea has a relatively high frequency component due to the discontinuity in the signal, hence, of apnea is higher than the one of the normal respiration. The experiments have been carried out using 250 respiration signals, and the status is picked randomly so that the proposed method can be reliably evaluated in quantitative way.

\section{Conclusion}

In this paper, respiratory status is classified in frequency domain. DFT is simply calculated and the procedures of spectral analysis is straightforward so that the proposed approach can be applied to estimation of vital signal using simple and low-cost device. The experimental results show that the proposed method can reliably classify the respiratory status, and it can be extended 
to development of the intelligent sensing system for the respiratory status. In the future, we will try to propos the approach to sensing respiratory status with short-time measurement in noninvasive way so that fast and efficient classification and abnormality detection can be accomplished.

\section{ACKNOWLEDGEMENTS}

This work was supported by Institute for Information \& communications Technology Promotion(IITP) grant funded by the Korea government(MSIT) (2016-0-00564, Development of Intelligent Interaction Technology Based on Context Awareness and Human Intention Understanding)

\section{REFERENCES}

[1] F. Q. AL-Khalidi, R. Saatchi, D. Burke, (2011) H. Elphick and S. Tan, "Respiration rate monitoring methods: A review", Pediatric Pulmonology, 46(6), 523-529.

[2] K. Watanabe, T. Watanabe, H. Watanabe, H. Ando, T. Ishikawa and K. Kobayashi, (2005) "Noninvasive measurement of heartbeat, respiration, snoring and body movements of a subject in bed via a pneumatic method", IEEE Transactions on Biomedical Engineering, 52(12), 2100-2107.

[3] A. D. Droitcour, T. B. Seto, B-K. Park, S. Yamada, A. Vergara, C. Hourani, T. Shing, A. Yuen, V. Lubecke and O. Boric-Lubecke, (2009) "Non-contact respiratory rate measurement validation for hospitalized patients", 2009 Annual International Conference of the IEEE Engineering in Medicine and Biology Society, pp. 4812-4815. Minneapolis, MN, USA.

[4] M. A. Cretikos, R. Bellomo, K. Hillman, J. Chen, S. Finfer and A. Flabouris, (2008) "Respiratory rate: the neglected vital sign", Medical Journal of Australia, 188(11),657-659.

[5] G. Ossberger, T. Buchegger, E. Schimback, A. Stelzer and R. Weigel, (2004) "Non-invasive respiratory movement detection and monitoring of hidden humans using ultra wideband pulse radar", 2004 International Workshop on Ultra Wideband Systems Joint with Conference on Ultra Wideband Systems and Technologies. Joint UWBST \& IWUWBS 2004 (IEEE Cat. No.04EX812), Kyoto, Japan, pp. 395-399.

[6] K. S. Tan, R. Saatchi, H. Elphick and D. Burke, (2010) "Real-time vision based respiration monitoring system", 7th International Symposium on Communication Systems, Networks \& Digital Signal Processing (CSNDSP 2010), Newcastle upon Tyne, UK, pp. 770-774.

[7] J. Chen, P. Jönsson, M. Tamura, Z. Gu, B. Matsushita and L. Eklundh, (2004) "A simple method for reconstructing a high-quality NDVI time-series data set based on the Savitzky-Golay filter", Remote Sensing Environment, 91(3-4), 332-344.

[8] S. Kim, C. Hwang, J. Kim, C. Park and D. Lee, (2018) "Application to Detection and Classification of Respiratory Status based on a Signal Correlation", to appear In: Annual Fall Conference of the Korean Institute of Electronics and Information Engineers (IEIE).

[9] S. Kim, C. Hwang, J. Kim and D. Lee, (2019) "Signal Classification based System for Monitoring Abnormal Respiratory Status", submitted to Journal of the Institute of Electronics and Information Engineers. 


\section{Authors}

\section{Suyeol Kim}

Suyeol Kim is in department of biomedical engineering, Keimyung University, Daegu, Republic of Korea. He is currently working on signal processing, particularly on respiration signal analysis. He is currently pursuing his bachelor degree in engineering.

\section{Chaehwan Hwang}

Chaehwan Hwang is in department of biomedical engineering, Keimyung University, Daegu, Republic of Korea. He is currently working on signal processing, particularly on respiration signal analysis in frequency domain. He is currently pursuing his bachelor degree in engineering.

\section{Jisu Kim}

Jisu Kim is in department of computer engineering, Keimyung University, Daegu, Republic of Korea. He is currently working on image processing, computer vision, signal processing and machine learning. He is currently pursuing his M.S degree in computer engineering.

\section{Cheolhyeong Park}

Cheolhyeong Park is in department of computer engineering, Keimyung University, Daegu, Republic of Korea. He is currently working on geometric image analysis, computer vision, computer graphics and machine learning. $\mathrm{He}$ is in the course of integrated B.S and M.S degree in computer engineering.

\section{Deokwoo Lee}

Dr. Deokwoo Lee is an Assistant Professor in the department of computer engineering at Keimyung University. Dr. Lee has received B.S degree in electrical engineering from Kyungpook National University, Daegu, Republic of Korea, and M.S and Ph.D degree from North Carolina State University, Raleigh, NC, USA, respectively. He has been working on the areas of computer vision, image processing, signal processing and machine learning. In particular, he has been conducting camera calibration, bio-signal analysis and image denoising 